\title{
Anesthetic Management of An Adolescent With Lowe Syndrome During Posterior Spinal Fusion
}

\author{
Badar Malik $^{\mathrm{a}}$, Hina Walia ${ }^{\mathrm{b}, \mathrm{d}}$, David P. Martin ${ }^{\mathrm{b}, \mathrm{c}}$, \\ Joseph D. Tobias ${ }^{b, c}$
}

\begin{abstract}
Lowe syndrome, also known as oculocerebrorenal syndrome, is a rare $\mathrm{X}$-linked disorder that was first described in 1952. Associated endorgan involvement includes the eye, central nervous system (CNS), and the kidney with proximal renal tubular acidosis. Life expectancy is reduced to mid-adulthood as a result of renal disease, hypotonia, and an increased susceptibility to infections. Given the multi-system involvement, surgical intervention may be required to address the sequelae of the disease process. We present a 16-year-old boy with Lowe syndrome who required anesthetic care for posterior spinal fusion for the treatment of neuromuscular scoliosis. Previous reports of anesthetic care of these patients are reviewed, end-organ involvement is discussed, and options for anesthetic care are presented.
\end{abstract}

Keywords: Lowe syndrome; Oculocerebrorenal syndrome; Posterior spinal fusion

\section{Introduction}

Lowe syndrome, also known as oculocerebrorenal syndrome, is a rare $\mathrm{X}$-linked $(\mathrm{Xq} 25-\mathrm{q} 26)$ disorder that was first described by Lowe and colleagues in 1952 [1]. It has an estimated prevalence of 1 in 500,000 patients. The OCRL1 gene that codes for phosphatidylinositol 4,5-bisphosphate 5-phosphatase enzyme is defective, which leads to the accumulation phosphatidylinositol 4,5-bisphosphate in multiple subcellular compartments $[2,3]$. The phenotypic expression may vary somewhat based on the specific mutation, but end-organ involvement generally includes the eye, central nervous system (CNS), and the kidney with proximal renal tubular acidosis [4]. Ocular manifestations

Manuscript submitted October 31, 2017, accepted November 3, 2017

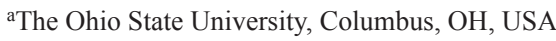

bDepartment of Anesthesiology \& Pain Medicine, Nationwide Children's Hospital, Columbus, $\mathrm{OH}$, USA

'Department of Anesthesiology, The Ohio State University, Columbus, OH, USA

${ }^{\mathrm{d} C}$ Corresponding Author: Hina Walia, Department of Anesthesiology \& Pain Medicine, Nationwide Children's Hospital, 700 Children's Drive, Columbus, OH 43205, USA. Email: Hina.Walia@nationwidechildrens.org

doi: https://doi.org/10.14740/jmc2949w including cataracts and severe muscular hypotonia generally present early in life and may be apparent at birth. Renal manifestations including Fanconi syndrome often develop later. Life expectancy is reduced to mid-adulthood as a result of renal disease, CNS involvement, and an increased susceptibility to infections [4]. Given the multi-system involvement, surgical intervention may be required to address the sequelae of the disease process. We present a 16-year-old boy with Lowe syndrome who required anesthetic care for posterior spinal fusion for the treatment of neuromuscular scoliosis. Previous reports of anesthetic care of these patients are reviewed, end-organ involvement is discussed, and options for anesthetic care are presented.

\section{Case Report}

Institutional Review Board approval is not required at Nationwide Children's Hospital (Columbus, Ohio) for the presentation of single case reports. The patient was a 16-year-old, 31.2 $\mathrm{kg}$ adolescent who presented for posterior spinal fusion with instrumentation for the treatment of neuromuscular scoliosis associated with Lowe syndrome. He was born at 40 weeks gestation and there were no immediate perinatal complications. By 1 year of age, he presented for evaluation due to hypotonia and poor weight gain with the subsequent diagnosis of Lowe syndrome based on clinical presentation and laboratory evaluation. The patient's motor and speech skills were delayed. He did not ambulate until 2.5 years of age. His past medical history was significant for Lowe syndrome, associated renal involvement with Fanconi syndrome, cataracts, hypertension, intellectual disability, short stature, and osteopenia with a history of previous femur fractures. Past surgical history included orthopedic surgery for the femur fractures, tear duct surgery, and a kidney biopsy at the age of 16 years. Allergies were listed as codeine and gentamicin. Maternal history was significant for hypertension and there was a family history of two maternal uncles with Lowe syndrome. Current medications include growth hormone $(1.6 \mathrm{mg}$ subcutaneous injection every night) for short stature, amlodipine (5 $\mathrm{mg}$ oral tablet by mouth once daily) for hypertension, potassium citrate, sodium citrate, and ergocalciferol. Physical examination revealed mild retrognathia, a diminished thyromental distance and a Mallampati III airway. Cardiovascular and pulmonary examinations were normal with regular rate and rhythm without a murmur. Preoperative laboratory evaluation including electrolytes, renal func- 
tion, coagulation function, blood glucose and hepatic function were normal except for a low serum potassium $(3.2 \mathrm{mEq} / \mathrm{L})$ and a low serum bicarbonate $(18 \mathrm{mEq} / \mathrm{L})$. The patient was held nil per os for $8 \mathrm{~h}$ prior to surgery and was transported to the operating room where routine American Society of Anesthesiologists' monitors were applied. An 18 gauge intravenous cannula was placed in the right hand and midazolam $(2 \mathrm{mg})$ administered intravenously prior to anesthetic induction. Anesthesia was induced with propofol (100 mg). Bag-valve-mask ventilation was provided without difficulty. Neuromuscular blockade for endotracheal intubation was provided with rocuronium (15 $\mathrm{mg}$ ) and direct laryngoscopy was performed with a Macintosh 3 laryngoscope blade which was unsuccessful with poor visualization of the glottis and a Cormack-Lehane grade III view. A second laryngoscopy performed with a Miller 2 blade with cricoid pressure revealed a Cormack-Lehane grade II view and a $6.0 \mathrm{~mm}$ cuffed endotracheal tube was placed. A 22 gauge arterial cannula was placed in the right radial artery and a second intravenous cannula was placed in the left arm. Tranexamic acid was administered for the prevention of fibrinolysis and to limit intraoperative blood loss $(50 \mathrm{mg} / \mathrm{kg}$ bolus dose followed by an infusion at $5 \mathrm{mg} / \mathrm{kg} / \mathrm{h}$ ). Per our usual practice to allow for neurophysiological monitoring during spinal surgery, anesthesia was maintained with desflurane titrated to maintain the bispectral index (BIS) at 50 - 60 and a sufentanil infusion of $0.1-0.3 \mu \mathrm{g} / \mathrm{kg} / \mathrm{h}$ [5]. Clevidipine was added to maintain the mean arterial pressure at $55-70 \mathrm{~mm} \mathrm{Hg}$ to limit intraoperative blood loss. Methadone $(0.15 \mathrm{mg} / \mathrm{kg})$ was administered to provide postoperative analgesia. Baseline neurophysiological monitoring including motor evoked potentials (MEPs) and somatosensory evoked potentials (SSEPs) were obtained. The patient was turned and positioned prone on the Jackson table using a prone pillow. Intraoperative heart rate varied from 60 - 100 beats/min with a normal sinus rhythm. No allogeneic blood products were administered. The estimated blood loss was $650 \mathrm{~mL}$. Total fluids administered during the procedure were $1,100 \mathrm{~mL}$ of $5 \%$ albumin and Normosol-R ${ }^{\circledR}$. Sodium bicarbonate, potassium chloride, and calcium chloride were administered based on intraoperative arterial blood gas and electrolyte values. During wound closure, the sufentanil and tranexamic acid infusions were discontinued. Residual neuromuscular blockade was reversed with sugammadex. Acetaminophen $(15 \mathrm{mg} / \mathrm{kg})$ was administered intravenously along with incremental doses of hydromorphone to provide postoperative analgesia. Following completion of the surgical procedure, the patient was turned supine and his trachea was extubated when awake. The patient was transferred to the post anesthesia care unit (PACU) and then admitted to the inpatient surgical ward with continuous pulse oximetry monitoring. Postoperative pain control was provided with hydromorphone delivered via nurse-controlled analgesia. The remainder of his postoperative course was uncomplicated and he was discharged home on postoperative day 5 .

\section{Discussion}

The classic form of the oculocerebrorenal syndrome is char- acterized by the triad of congenital cataracts, neonatal or infantile hypotonia followed by severe intellectual impairment, and renal tubular dysfunction with slowly progressive renal failure. Effective perioperative care begins with a thorough preoperative evaluation including an evaluation of end-organ impact of the primary disease process affected by Lowe syndrome. Various skeletal problems related to osteopenia from hypophosphatemic rickets, retrognathism, high arched palate, craniofacial abnormalities, and abnormal teeth may impact airway management including direct laryngoscopy and tracheal intubation. Previous reports have anecdotally noted difficulties with endotracheal intubation $[6,7]$. In our patient, physical examination suggested the potential for difficulties with endotracheal intubation as there was retrognathism and a short thyromental distance. Although bas-valve-mask ventilation was uncomplicated, a second attempt at laryngoscopy with a Miller instead of a Macintosh blade along with cricoid pressure was necessary for successful endotracheal intubation. Given these concerns, the ability to accomplish adequate bagvalve mask ventilation should be demonstrated prior to the use of neuromuscular blocking agents. Additionally, the appropriate equipment for dealing with the difficult airway should be readily available including indirect vidoelaryngoscopy $[8,9]$.

One of the primary components of Lowe syndrome is renal involvement manifested initially as Fanconi syndrome. Although the severity of renal disease varies from patient to patient and progresses with age, symptoms include aminoaciduria, hypercalciuria, low molecular weight (LMW) proteinuria, renal phosphate and bicarbonate wasting, and acidosis [4, $10,11]$. Initial symptoms present during the first months of life. Many patients develop chronic renal failure during the second decade of life, which can lead to end-stage renal disease (ESRD) requiring hemodialysis. Given the invariable effect on electrolytes and acid-base status, preoperative and intraoperative monitoring of $\mathrm{pH}$ and electrolytes (especially potassium) is recommended. Intraoperative supplementation with sodium bicarbonate, calcium chloride, and potassium chloride, may be needed to correct acidosis and electrolyte disturbances. In our patient, we used an isotonic intravenous fluid (Normosol$\mathrm{R}^{\circledR}$ ) which contains buffer (acetate and gluconate) to limit the need for sodium bicarbonate administration. Additional renal involvement includes a concentrating defect with an inability to conserve water with isosthenuria. As such, strict perioperative precautions to prevent dehydration are recommended with the additional caveat that urine output may not be an accurate monitor of intravascular volume status.

Renal tubular dysfunction with chronic bicarbonate loss results in a chronic metabolic acidosis. Chronic metabolic acidosis may potentiate the depressant effects of intravenous and volatile anesthetic agents on myocardial function as well as depress the response to vasoactive agents. As opioids are weak bases, metabolic acidosis increases the unionized fraction thereby facilitated penetration into the CNS with the possibility of potentiating opioid-induced respiratory depression. These concerns must be addressed postoperatively and ongoing postoperative monitoring of respiratory status is recommended for patients receiving opioids.

In addition to chronic metabolic acidosis, electrolyte disturbances including hypokalemia, hypophosphatemia, hy- 
Table 1. Previous Reports of Anesthetic Care for Patients With Lowe Syndrome

\begin{tabular}{|c|c|c|}
\hline Author and reference number & Patient demographics & Findings \\
\hline Panday et al [6] & $\begin{array}{l}\text { 4-month-old, } 5 \mathrm{~kg} \text { boy } \\
\text { scheduled for cataract surgery. }\end{array}$ & $\begin{array}{l}\text { Preoperative electrolytes imbalances were corrected with potassium, } \\
\text { calcium, magnesium, phosphate, and bicarbonate supplementation. } \\
\text { Anesthetic induction with halothane, fentanyl and thiopental. } \\
\text { Endotracheal intubation was difficult, accomplished on the fourth } \\
\text { attempted with a Cormack-Lehane grade } 3 \text { view. Maintenance anesthesia } \\
\text { with isoflurane, nitrous oxide fentanyl, and atracurium. No perioperative } \\
\text { complications noted. }\end{array}$ \\
\hline Komatsu et al [19] & $\begin{array}{l}\text { 7-month-old, } 6.9 \mathrm{~kg} \text { boy for } \\
\text { cataract and glaucoma surgery. }\end{array}$ & Anesthetic induction and maintenance with sevoflurane in nitrous oxide. \\
\hline
\end{tabular}

pomagnesemia, and hypocalcemia may be seen. Extreme hypokalemia may potentiate cardiac arrhythmias. Hypokalemia may also increase the sensitivity to non-depolarizing neuromuscular blocking agents. Dosing should be titrated using the train-of-four and doses decreased accordingly (see below). Although chronic hypokalemia is generally well tolerated, replacement therapy may be required when serum potassium levels are less than $2.5 \mathrm{mEq} / \mathrm{L}$. Hyperventilation should be avoided to prevent a similar intracellular shift of potassium. Serum glucose should be monitored and dextrose-containing solutions limited, because hyperglycemia and secondary insulin release may promote the intracellular movement of potassium and further lower serum potassium [7]. This concern must be balanced with the potential for hypoglycemia. Loss of L-carnitine is associated with a decreased ability to tolerate fasting with the potential for hypoglycemia [7].

Hypotonia is also commonly seen in patients with Lowe syndrome and may increase the incidence of postoperative respiratory insufficiency related to upper airway obstruction or respiratory impairment. Given the potential impact of long acting anesthetic agents on postoperative respiratory function, we would recommend the use of short acting agents. The combination of desflurane and sufentanil were chosen in our patient based on our usual clinical practice to facilitate neurophysiological monitoring. These agents also provide for the rapid return of function to baseline with limited residual effects during the immediate postoperative period [5]. Depth of anesthesia monitoring with the bispectral index was used to titrate the desflurane to the desired level in a patient with neurocognitive dysfunction $[12,13]$.

Hypotonia may also impact the choice of neuromuscular blocking agent. Although there are limited data and clinical experience on which to make firm clinical recommendations, given the associated hypotonia, the use of succinylcholine is controversial and relatively contraindicated because of the potential for hyperkalemia [14]. Anecdotal experience has noted elevated creatinine phosphokinase (CPK) levels in some patients suggested primary muscle involvement. Hypotonia may lead to an exaggerated response to neuromuscular blocking agents (NMBAs), making precise dosing with train-of-four monitoring a prerequisite to avoid prolonged recovery times. Residual neuromuscular blockade should be reversed at the completion of the procedure and full recovery documented prior to tracheal extubation. Reversal with suggamadex may offer an advantage in patients with pre-existing hypotonia and limit the risk of residual neuromuscular blockade [15]. Regardless of the anesthetic agents chosen, continuous monitoring of postoperative respiratory function is suggested, especially when opioids are administered.

Given the progressive deterioration of CNS function, seizures are a frequent co-morbid condition in patients with Lowe syndrome [16]. The dosing of anticonvulsant medications should be optimized preoperatively and administered the morning of the procedure despite the patient's nil per os status. Subsequent intraoperative dosing is continued as needed. Consultation with the neurology or pharmacology service may be helpful to determine dosing conversion from enteral to intravenous administration or to guide intraoperative dosing. In general clinical practice, there does not appear to be any specific contraindication or advantage of specific anesthetic agents in patients with underlying seizure disorders.

Other issues to be considered include the potential for intraoperative hypothermia related to poor CNS temperature regulation. Warming of the operating room and intravenous fluids as well as forced air warming during the procedure are generally effective measures. Ocular involvement includes glaucoma mandating close attention to intraoperative eye care and avoidance of direct external pressure. As noted above, 
Table 2. Perioperative Concerns of Lowe Syndrome

\begin{tabular}{l} 
1. Difficult airway management \\
2. Proximal renal tubular dysfunction \\
a. metabolic acidosis \\
b. hypokalemia \\
c. hypocalcemia \\
d. hypophosphatemia \\
e. hypomagnesemia \\
f. hypercalciuria with stone formation \\
3. Renal insufficiency \\
4. Renal concentrating defect (isosthenuria) \\
5. Ocular involvement \\
a. cataracts \\
b. glaucoma \\
6. Central nervous system involvement \\
a. developmental delay \\
b. seizures \\
7. Bone fragility due to hypophosphatemic rickets \\
8. Hypoglycemia \\
9. Hypotonia \\
10. Platelet aggregation defect - generally of limited clinical \\
significance \\
\hline
\end{tabular}

chronic calcium and phosphate losses in the urine leads to osteopenia and the potential for fractures, necessitating care during positioning. In some patients, a platelet aggregation defect has been reported [17]. The clinical significance is generally limited and the defect can be corrected by the administration of tranexamic acid.

In summary, we discuss the perioperative care of patient Lowe syndrome, an X-linked disorder often associated with renal involvement with Fanconi syndrome, ocular involvement including cataracts and glaucoma, as well as central nervous system with seizures and developmental delay. To date, there are a limited number of reports in the English-language literature outlining the perioperative care of these patients (Table 1) $[6,7,18,19]$. Specific perioperative concerns include the potential for difficulties with airway management, bone fragility with positioning issues, electrolyte disturbances, chronic metabolic acidosis, and hypotonia (Table 2).

\section{Conflict of Interest}

None.

\section{References}

1. Lowe CU, Terrey M, Mac LE. Organic-aciduria, decreased renal ammonia production, hydrophthalmos, and mental retardation; a clinical entity. AMA Am J Dis Child. 1952;83(2):164-184.

2. Mehta ZB, Pietka G, Lowe M. The cellular and physiological functions of the Lowe syndrome protein OCRL1. Traffic. 2014;15(5):471-487.

3. Attree O, Olivos IM, Okabe I, Bailey LC, Nelson DL, Lewis RA, McInnes RR, et al. The Lowe's oculocerebrorenal syndrome gene encodes a protein highly homologous to inositol polyphosphate-5-phosphatase. Nature. 1992;358(6383):239-242.

4. Bokenkamp A, Ludwig M. The oculocerebrorenal syndrome of Lowe: an update. Pediatr Nephrol. 2016;31(12):2201-2212.

5. Martin DP, Bhalla T, Thung A, Rice J, Beebe A, Samora W, Klamar J, et al. A preliminary study of volatile agents or total intravenous anesthesia for neurophysiological monitoring during posterior spinal fusion in adolescents with idiopathic scoliosis. Spine (Phila Pa 1976). 2014;39(22):E1318-1324.

6. Pandey R, Garg R, Chakravarty C, Darlong V, Punj J, Chandralekha. Lowe's syndrome with Fanconi syndrome for ocular surgery: perioperative anesthetic considerations. J Clin Anesth. 2010;22(8):635-637.

7. Saricaoglu F, Demirtas F, Aypar U. Preoperative and perioperative management of a patient with Lowe syndrome diagnosed to have Fanconi's syndrome. Paediatr Anaesth. 2004;14(6):530-532.

8. Engelhardt T, Weiss M. A child with a difficult airway: what do I do next? Curr Opin Anaesthesiol. 2012;25(3):326-332.

9. Bryant J, Krishna SG, Tobias JD. The difficult airway in pediatrics. Advan Anesth 2013;31:31-60.

10. Richards W, Donnell GN, Wilson A, Stowens D, Perry T. The oculocerebrorenal syndrome of Lowe. Am J Dis Child. 1965;109;185-203.

11. Haque SK, Ariceta G, Batlle D. Proximal renal tubular acidosis: a not so rare disorder of multiple etiologies. Nephrol Dial Transplant. 2012;27(12):4273-4287.

12. Zhang JM, Wang F, Xin Z, Zi TT, Lv H. Treatment of different-aged children under bispectral index monitoring with intravenous anesthesia with propofol and remifentanil. Eur Rev Med Pharmacol Sci. 2015;19(1):64-69.

13. Choudhry DK, Brenn BR. Bispectral index monitoring: a comparison between normal children and children with quadriplegic cerebral palsy. Anesth Analg. 2002;95(6):1582-1585.

14. Martyn JA, Richtsfeld M. Succinylcholine-induced hyperkalemia in acquired pathologic states: etiologic factors and molecular mechanisms. Anesthesiology. 2006;104(1):158-169.

15. Tobias JD. Current evidence for the use of sugammadex in children. Paediatr Anaesth. 2017;27(2):118-125.

16. Charnas L. Seizures in the oculocerebrorenal syndrome of Lowe. Neurology. 1989;39;216.

17. Lasne D, Baujat G, Mirault T, Lunardi J, Grelac F, Egot $\mathrm{M}$, Salomon R, et al. Bleeding disorders in Lowe syndrome patients: evidence for a link between OCRL mutations and primary haemostasis disorders. Br J Haematol. 2010;150(6):685-688. 
18. Ramanathan G, Patil S. A case of Lowe syndrome (oculocerebrorenal syndrome): Clinical implications and anaesthetic management. S Afr J Anaesth Analg. 2009;15;29-31.
19. Komatsu H, Sakakibara M, Yoshimura Y, Kinoshita H, Yokono S, Ogli K. Anesthetic management for a patient with oculocerebrorenal (Lowe's) syndrome. J Anesth. 1994;8(1):121-123. 